\title{
BETWEEN THE PROBLEMS OF PÓLYA AND TURÁN
}

\author{
MICHAEL J. MOSSINGHOFF ${ }^{\bowtie}$ and TIMOTHY S. TRUDGIAN
}

(Received 6 May 2011; accepted 1 February 2012; first published online 27 September 2012)

Communicated by I. E. Shparlinski

Dedicated to the memory of Alf van der Poorten

\begin{abstract}
We investigate the behaviour of the function $L_{\alpha}(x)=\sum_{n \leq x} \lambda(n) / n^{\alpha}$, where $\lambda(n)$ is the Liouville function and $\alpha$ is a real parameter. The case where $\alpha=0$ was investigated by Pólya; the case $\alpha=1$, by Turán. The question of the existence of sign changes in both of these cases is related to the Riemann hypothesis. Using both analytic and computational methods, we investigate similar problems for the more general family $L_{\alpha}(x)$, where $0 \leq \alpha \leq 1$, and their relationship to the Riemann hypothesis and other properties of the zeros of the Riemann zeta function. The case where $\alpha=1 / 2$ is of particular interest.
\end{abstract}

2010 Mathematics subject classification: primary 11M26; secondary 11M45, 11N64, 11 Y35.

Keywords and phrases: Liouville function, Riemann hypothesis, Pólya's problem, Turán's problem.

\section{Introduction}

Let $\lambda(n)$ denote the Liouville function, the completely multiplicative arithmetic function determined by $\lambda(p)=-1$ for each prime $p$. It is well known (see [10, p. 6]) that

$$
\sum_{n=1}^{\infty} \frac{\lambda(n)}{n^{s}}=\frac{\zeta(2 s)}{\zeta(s)}
$$

if $\sigma>1$. (We follow the usual convention that $s=\sigma+i t$ with $\sigma$ and $t$ real throughout this paper.) The Liouville function arises in a natural way in many problems in analytic number theory.

In 1919, Pólya [7] studied the function $L(x)=\sum_{n \leq x} \lambda(n)$, proving that if $p$ is a prime greater than 7 and $\mathbb{Q}(\sqrt{-p})$ has class number 1 , then $L((p-3) / 4)=0$. He also computed the values of $L(x)$ for $x$ up to approximately 1500, noting all zeros that occurred, and remarked that $L(x)$ is never positive across this range (after $x=1$ ).

This work was partially supported by a grant from the Simons Foundation (\#210069 to M. Mossinghoff).

(C) 2012 Australian Mathematical Publishing Association Inc. 1446-7887/2012 \$16.00 
In addition, Pólya remarked that the Riemann hypothesis would follow if one could establish that $L(x)$ eventually has constant sign. The assertion that $L(x)$ is never positive for $x \geq 2$ is often called 'Pólya's conjecture' in the literature, although it appears that Pólya never in fact stated this as a conjecture, at least not in print. We label an inverted version here as Pólya's problem.

Pólya's Problem. Show that $L(x)$ changes sign infinitely often, and determine the smallest $x \geq 2$ where $L(x)>0$.

In 1942, Ingham [4] noted that if $L(n)$ is eventually of constant sign, then not only would the Riemann hypothesis follow, but also the zeros of $\zeta(s)$ would all be simple, and further there would exist infinitely many linear dependencies over $\mathbb{Z}$ of the imaginary parts of the zeros of $\zeta(s)$ in the upper half plane.

In 1948, Turán [11] investigated some properties of a weighted sum involving the Liouville function. Let $T(x)=\sum_{n \leq x} \lambda(n) / n$. In connection with some studies of partial sums of the Riemann zeta function, Turán showed that if there exists a positive constant $c$ such that $T(n)>-c / \sqrt{n}$ for all sufficiently large $n$, then the Riemann hypothesis would follow. He also reported that several assistants had verified that $T(n)>0$ for $n \leq 1000$. The statement that $T(n)>0$ for all $n$ is often called 'Turán's conjecture,' but, as with Pólya's problem, Turán never stated this as a formal conjecture. (In fact, Turán took issue with the term in a later paper [12].) We label the inverted form here as Turán's problem.

Turań's Problem Show that $T(x)$ changes sign infinitely often, and determine the smallest $x \geq 1$ where $T(x)<0$.

In 1958, Haselgrove [3] used the results of Ingham [4] to answer the first part of both problems, proving that both $L(x)$ and $T(x)$ change sign infinitely often. However, he did not determine any particular values where $L(x)>0$ or where $T(x)<0$. An explicit crossing point for $L(x)$ was first computed by Lehman in 1960 [5], who found that $L(906180359)=1$. The smallest $x$ (other than 1 ) where $L(x)>0$ was not determined until 1980, when Tanaka [9] showed that this occurs at $x=906105257$. An explicit crossing point for $T(x)$ was not discovered until recently. In 2008, Borwein, Ferguson, and the first author [1] determined that the smallest $x$ where $T(x)<0$ is $x=72185376951205$.

It is interesting that negative values appear to dominate in $L(x)$, while positive ones dominate in $T(x)$. In this paper, we explore this further by considering a natural generalisation of the problems of Pólya and Turán. For $\alpha \geq 0$, let

$$
L_{\alpha}(x)=\sum_{n \leq x} \frac{\lambda(n)}{n^{\alpha}},
$$

so that $L_{0}(x)=L(x)$ and $L_{1}(x)=T(x)$. If $\alpha>1$, then $L_{\alpha}(x)$ converges absolutely to $\zeta(2 \alpha) / \zeta(\alpha)>0$ by (1.1), and so only finitely many sign changes may occur in this situation. The cases where $0 \leq \alpha \leq 1$ are therefore those of interest in this paper. 
In this paper we show that the family of functions $L_{\alpha}(x)$ for $0 \leq \alpha \leq 1$ is closely tied to questions about the Riemann zeta function. In particular, we show that sign constancy in certain expressions involving $L_{\alpha}(x)$ for $\alpha$ in this range implies the Riemann hypothesis, as well as the simplicity of the zeros of the zeta function (see Theorem 2.4). We also prove that Ingham's result on the existence of linear dependencies among the zeros of the zeta function on the critical line has a natural analogue in this generalised setting (see Theorem 3.3). We then pose a natural generalisation of the problems of Pólya and Turán for $0 \leq \alpha \leq 1$, and present a similar problem for the case $\alpha=1 / 2$ which would imply the Riemann hypothesis and a bound on the multiplicity of the zeros, but does not appear to be connected to the linear dependence question. Finally, we report on some empirical investigations of these problems.

This paper is organised as follows. Section 2 establishes connections between the behaviour of $L_{\alpha}(x)$ and the Riemann hypothesis, and Section 3 generalises Ingham's results on the linear independence question. Section 4 discusses some open problems regarding sign changes in expressions involving $L_{\alpha}(x)$ for various $\alpha$, and Section 5 describes some computations regarding the functions $L_{\alpha}(x)$ for particular values of $\alpha$.

\section{2. $L_{\alpha}(x)$ and the Riemann hypothesis}

It is well known that the Riemann hypothesis is equivalent to the statement that $L_{0}(x)=O\left(x^{\frac{1}{2}+\epsilon}\right)$, and that the prime number theorem is equivalent to the statement that $\lim _{x \rightarrow \infty} L_{1}(x)=0$. We begin by extending these statements to the case of more general $\alpha$. The proofs of the following two results are similar to those of related statements involving the Möbius function found in [10, Section 14.25].

THeorem 2.1. If the Riemann hypothesis holds, then for all $\alpha \in(1 / 2,1]$ and all $\epsilon>0$,

$$
L_{\alpha}(x)=\frac{\zeta(2 \alpha)}{\zeta(\alpha)}+O\left(x^{\frac{1}{2}-\alpha+\epsilon}\right)
$$

Conversely, if

$$
\lim _{x \rightarrow \infty} L_{\alpha}(x)=\frac{\zeta(2 \alpha)}{\zeta(\alpha)}
$$

for all $\alpha \in(1 / 2,1]$, then the Riemann hypothesis is true.

Proof. We employ Perron's formula [10, Lemma 3.12] on the function

$$
f(s)=\sum_{n \geq 1} \frac{\lambda(n)}{n^{\alpha+s}}=\frac{\zeta(2(\alpha+s))}{\zeta(\alpha+s)}
$$

for $\sigma>1-\alpha$, to obtain some information on the functions $L_{\alpha}(x)$. We find that for any $\alpha \in[0,1]$,

$$
L_{\alpha}(x)=\frac{1}{2 \pi i} \int_{2-i T}^{2+i T} \frac{\zeta(2(\alpha+w))}{\zeta(\alpha+w)} \cdot \frac{x^{w}}{w} d w+O\left(\frac{x^{2}}{T}\right)
$$


Using (2.1), we see that

$$
\begin{aligned}
L_{\alpha}(x)=\frac{1}{2 \pi i} & \left(\int_{2-i T}^{\frac{1}{2}-\alpha+\delta-i T}+\int_{\frac{1}{2}-\alpha+\delta-i T}^{\frac{1}{2}-\alpha+\delta+i T}+\int_{\frac{1}{2}-\alpha+\delta+i T}^{2+i T}\right) \frac{\zeta(2(\alpha+w))}{\zeta(\alpha+w)} \cdot \frac{x^{w}}{w} d w \\
& +\frac{\zeta(2 \alpha)}{\zeta(\alpha)}+O\left(\frac{x^{2}}{T}\right),
\end{aligned}
$$

where $0<\delta<\alpha-1 / 2$. If the Riemann hypothesis holds, then $\zeta(\sigma+i t)=O\left(t^{\epsilon}\right)$ and $1 / \zeta(\sigma+i t)=O\left(t^{\epsilon}\right)$, so the first and third integrals above are $O\left(T^{-1+\epsilon} x^{2}\right)$, and the second is $O\left(x^{\frac{1}{2}-\alpha+\delta} \int_{-T}^{T}(1+|t|)^{-1+\epsilon} d t\right)=O\left(x^{\frac{1}{2}-\alpha+\delta} T^{\epsilon}\right)$. Consequently,

$$
L_{\alpha}(x)=\frac{\zeta(2 \alpha)}{\zeta(\alpha)}+O\left(x^{2} T^{-1+\epsilon}\right)+O\left(x^{\frac{1}{2}-\alpha+\delta} T^{\epsilon}\right),
$$

and choosing $T=x^{3}$ produces the required estimate. Conversely, if $L_{\alpha}(x)$ converges for $\alpha>1 / 2$, then $L_{s}(x)$ converges uniformly in the half plane $\sigma \geq \sigma_{0}>1 / 2$, and thus represents an analytic function in this region. The limit function is certainly $\zeta(2 s) / \zeta(s)$ when $\sigma>1$, as one may verify easily by using the Euler product representation for the zeta function in this region, and so this must also be the case for $\sigma>1 / 2$. Thus, the Riemann hypothesis follows.

Theorem 2.2. If the Riemann hypothesis holds, then for all $\alpha \in[0,1 / 2]$ and all $\epsilon>0$,

$$
L_{\alpha}(x)=O\left(x^{\frac{1}{2}-\alpha+\epsilon}\right) .
$$

Conversely, if this estimate holds for some $\alpha \in[0,1 / 2]$, then the Riemann hypothesis is true.

Proof. Let $\alpha \in[0,1 / 2]$ and $\epsilon>0$. Using Perron's formula as in the proof of Theorem 2.1,

$$
L_{\alpha}(x)=O\left(x^{2} T^{-1+\epsilon}\right)+O\left(x^{\frac{1}{2}-\alpha+\epsilon} T^{\epsilon}\right)
$$

under the assumption of the Riemann hypothesis, since the path of integration does not surround the origin, nor the poles on the $\frac{1}{2}-\alpha$ line. Selecting $T=x^{2}$ then produces (2.2). For the converse, if $0 \leq \alpha \leq 1 / 2$ and (2.2) holds, then by partial summation

$$
\lim _{x \rightarrow \infty} L_{s}(x)=(s-\alpha) \int_{1}^{\infty} L_{\alpha}(t) t^{-(s+1-\alpha)} d t
$$

for $\sigma>1 / 2$, and so $L_{s}(x)$ converges for $\sigma>1 / 2$, and the Riemann hypothesis follows by Theorem 2.1 .

Ingham [4] proved that constancy in sign of $L_{0}(x)$ implies both the Riemann hypothesis and the simplicity of the zeros of the zeta function. In fact, he proved that these results follow under the weaker hypothesis that $L_{0}\left(e^{u}\right) e^{u / 2}$ is either bounded above by a constant for all $u$, or bounded below by a constant. We adapt his argument here to the more general case. We first define several required functions. 
First, for $0 \leq \alpha \leq 1$, define $\mathcal{L}_{\alpha}(x)$ by

$$
\mathcal{L}_{\alpha}(x)= \begin{cases}L_{\alpha}(x) & \text { if } 0 \leq \alpha<1 / 2 \text { or } \alpha=1, \\ L_{\alpha}(x)-\frac{\log x}{2 \zeta(1 / 2)} & \text { if } \alpha=1 / 2, \\ L_{\alpha}(x)-\frac{\zeta(2 \alpha)}{\zeta(\alpha)} & \text { if } 1 / 2<\alpha<1 .\end{cases}
$$

Next, define

$$
A_{\alpha}(u)=\mathcal{L}_{\alpha}\left(e^{u}\right) e^{\left(\alpha-\frac{1}{2}\right) u}
$$

for $u \geq 0$, and for complex $s$ set

$$
f_{\alpha}(s)=\frac{\zeta(1+2 s)}{(s-\alpha+1 / 2) \zeta(s+1 / 2)} .
$$

Finally, let

$$
F_{\alpha}(s)= \begin{cases}f_{\alpha}(s) & \text { if } 0 \leq \alpha<1 / 2 \text { or } \alpha=1, \\ f_{\alpha}(s)-\frac{1}{2 \zeta(1 / 2) s^{2}} & \text { if } \alpha=1 / 2, \\ f_{\alpha}(s)-\frac{\zeta(2 \alpha)}{\zeta(\alpha)(s-\alpha+1 / 2)} & \text { if } 1 / 2<\alpha<1 .\end{cases}
$$

Before stating a generalisation of Ingham's result for $L_{0}\left(e^{u}\right) e^{u / 2}$, we show that $F_{\alpha}$ is the Laplace transform of $A_{\alpha}$.

Lemma 2.3. Let $\alpha \in[0,1]$. With $F_{\alpha}(s)$ and $A_{\alpha}(u)$ defined as above,

$$
F_{\alpha}(s)=\int_{0}^{\infty} A_{\alpha}(u) e^{-s u} d u,
$$

and this integral converges for $\sigma>1 / 2$. Further, under the Riemann hypothesis, it converges for $\sigma>0$.

Proof. We use partial summation on (1.1) to write

$$
\frac{\zeta(2 s)}{\zeta(s)}=(s-\alpha) \int_{1}^{\infty} \mathcal{L}_{\alpha}(t) t^{-(s+1-\alpha)} d t .
$$

Suppose first that $0 \leq \alpha<1 / 2$ or $\alpha=1$. Since $|\lambda(n)|=1$, one easily obtains the trivial estimate $\mathcal{L}_{\alpha}(t)=L_{\alpha}(t)=O\left(t^{1-\alpha+\epsilon}\right)$ for any $\alpha \in[0,1]$, and so (2.7) certainly converges for $\sigma>1$. By Theorem 2.2, $\mathcal{L}_{\alpha}(t)=O\left(t^{\frac{1}{2}-\alpha+\epsilon}\right)$, assuming the Riemann hypothesis, and so (2.7) converges for $\sigma>1 / 2$ under this assumption. For $1 / 2<\alpha<1$, we begin by noting that

$$
\frac{\zeta(2 s)}{\zeta(s)}-\frac{\zeta(2 \alpha)}{\zeta(\alpha)}=(s-\alpha) \int_{1}^{\infty} \mathcal{L}_{\alpha}(t) t^{-(s+1-\alpha)} d t
$$


for $\sigma>1$, and, using Theorem 2.1, we see that this integral converges for $\sigma>1 / 2$ under the Riemann hypothesis. Finally, for the case $\alpha=1 / 2$, we see that

$$
\frac{\zeta(2 s)}{\zeta(s)}-\frac{1}{(2 s-1) \zeta(1 / 2)}=\left(s-\frac{1}{2}\right) \int_{1}^{\infty} \mathcal{L}_{1 / 2}(t) t^{-(s+1 / 2)} d t
$$

for $\sigma>1$, and if the Riemann hypothesis holds, then $\mathcal{L}_{1 / 2}(t)=O\left(t^{\epsilon}\right)$ by Theorem 2.2, and so this integral converges for $\sigma>1 / 2$ under this assumption. In all three cases, employing the change of variable $t \mapsto e^{u}$ and replacing $s$ by $s+1 / 2$ in these expressions produces the required statement.

We may now prove the following theorem.

THEOREM 2.4. Suppose that $\alpha \in[0,1]$ is a fixed real number. If there exists a constant $C$ for which $A_{\alpha}(u)-C$ has constant sign for all sufficiently large $u$, then the Riemann hypothesis follows, and all the zeros of the zeta function are simple. In addition, if there exists a constant $C$ for which $L_{1 / 2}(x)-C$ has constant sign for all sufficiently large $x$, then the Riemann hypothesis follows, and each nontrivial zero of the zeta function has order at most two.

Proof. Suppose that $\alpha \in[0,1]$ is fixed, and suppose without loss of generality that there exists a constant $C$ for which $A_{\alpha}(u)<C$ for all $u \geq 0$. Define the function $G_{\alpha}(s)$ for $\sigma>1 / 2$ by

$$
G_{\alpha}(s)=\int_{0}^{\infty}\left(C-A_{\alpha}(u)\right) e^{-s u} d u=\frac{C}{s}-F_{\alpha}(s),
$$

where the last expression follows by Lemma 2.3. Since the integrand has constant sign, the domain in which (2.8) is valid may be extended to the left of $\sigma=1 / 2$, upon appeal to Landau's theorem [6, Exercise 2.5.19], to the first real singularity of $C / \sigma-F_{\alpha}(\sigma)$. Since there are no zeros of $\zeta(s)$ along the positive real axis, it follows from (2.4) and (2.5) that $F_{\alpha}(\sigma)$ has its first real singularity at $\sigma=0$. Thus (2.8) defines an analytic function for $\sigma>0$. This means that $G_{\alpha}(s)$, and hence $F_{\alpha}(s)$, is regular for $\sigma>0$. This implies the Riemann hypothesis. Furthermore,

$$
\left|G_{\alpha}(s)\right| \leq \frac{C}{\sigma}-F_{\alpha}(\sigma) .
$$

The function $F_{\alpha}(\sigma)$ has only simple poles as $\sigma \rightarrow 0^{+}$, whence the right-hand side of (2.9) is $O\left(\sigma^{-1}\right)$. This means that $G(s)$, and therefore $F(s)$, can have no multiple pole on $\sigma=0$, and thus that all the zeros $\frac{1}{2}+i \gamma_{n}$ of $\zeta(s)$ are simple.

For the second statement, if $L_{1 / 2}(x)-C$ has constant sign for all $x \geq 1$, then for $\sigma>1 / 2$,

$$
\int_{0}^{\infty}\left(C-L_{1 / 2}\left(e^{u}\right)\right) e^{-s u} d u=\frac{1}{2 \zeta(1 / 2) s^{2}}+\frac{C}{s}-F_{1 / 2}(s),
$$

and appealing to Landau's theorem again produces the Riemann hypothesis. However, the pole of order two at $s=0$ in $f_{1 / 2}(s)$ implies that (2.10) is $O\left(\sigma^{-2}\right)$ as $\sigma \rightarrow 0^{+}$, so no zeros of the zeta function on the critical line have multiplicity greater than two. 


\section{3. $L_{\alpha}(x)$ and linear independence of the zeros}

Throughout this section, we assume the Riemann hypothesis and the simplicity of the zeros of the zeta function. From (2.4) and (2.5), it is easily seen that $F_{\alpha}(s)$ has a simple pole at $s=0$ for $0 \leq \alpha \leq 1$. This function also has a simple pole at $s=i \gamma_{n}$ for $n= \pm 1, \pm 2, \ldots$, where $\gamma_{n}$ denotes the magnitude of the ordinate of the $n$th zero of $\zeta(s)$ on the critical line, and $\gamma_{-n}=-\gamma_{n}$. Further, $F_{\alpha}(s)$ has no other poles in the half plane $\sigma \geq 0$.

We compute the residue of $F_{\alpha}(s)$ at each of its poles in this half plane:

$$
\operatorname{Res}\left(F_{\alpha}, 0\right)= \begin{cases}\frac{1}{(1-2 \alpha) \zeta(1 / 2)} & \text { if } \alpha \in[0,1 / 2) \cup(1 / 2,1] \\ \frac{\gamma}{\zeta(1 / 2)}-\frac{\zeta^{\prime}(1 / 2)}{2 \zeta(1 / 2)^{2}} & \text { if } \alpha=1 / 2\end{cases}
$$

where $\gamma$ denotes Euler's constant, and

$$
\operatorname{Res}\left(F_{\alpha}, i \gamma_{n}\right)=\frac{\zeta\left(2 \rho_{n}\right)}{\left(\rho_{n}-\alpha\right) \zeta^{\prime}\left(\rho_{n}\right)}
$$

for $0 \leq \alpha \leq 1$. In [4, Theorem 2], Ingham established that the sum

$$
\sum_{n \geq 1}\left|\operatorname{Res}\left(F_{0}, i \gamma_{n}\right)\right|=\sum_{n \geq 1}\left|\frac{\zeta\left(2 \rho_{n}\right)}{\rho_{n} \zeta^{\prime}\left(\rho_{n}\right)}\right|
$$

diverges. (His proof of this is similar to the argument in Titchmarsh [10, Section 14.27] that $\sum_{n \geq 1} 1 /\left(\left|\rho_{n} \zeta^{\prime}\left(\rho_{n}\right)\right|\right)$ diverges.) Since $\left|\rho_{n}-\alpha\right|^{-1} \gg\left|\gamma_{n}\right|^{-1} \gg\left|\rho_{n}\right|^{-1}$, it follows by the comparison test that $\sum_{n \geq 1}\left|\operatorname{Res}\left(F_{\alpha}, i \gamma_{n}\right)\right|$ diverges as well for $0 \leq \alpha \leq 1$. We record this result in the following lemma.

Lemma 3.1. Assume the Riemann hypothesis and the simplicity of the zeros of the zeta function. If $0 \leq \alpha \leq 1$, then $\sum_{n \geq 1} \mid \operatorname{Res}\left(F_{\alpha}\right.$, i $\left.\gamma_{n}\right) \mid=\infty$.

We next generalise two principal results of Ingham [4]. Let $T$ be a fixed real number with $T>1$, and let $N=N(T)$ be the number of zeros $\rho=\frac{1}{2}+i \gamma$ of the zeta function with $0 \leq \gamma \leq T$. For $\alpha \in[0,1]$, define $F_{\alpha}^{*}(s)=F_{\alpha, T}^{*}(s)$ by

$$
F_{\alpha}^{*}(s)=\frac{\operatorname{Res}\left(F_{\alpha}, 0\right)}{s}+\sum_{|n|=1}^{N} \frac{\operatorname{Res}\left(F_{\alpha}, i \gamma_{n}\right)}{s-i \gamma_{n}},
$$

so that $F_{\alpha}(s)-F_{\alpha}^{*}(s)$ is regular in the region $\sigma \geq 0,-T \leq t \leq T$. Let $A_{\alpha}^{*}(u)=A_{\alpha, T}^{*}(u)$ be the inverse Laplace transform of $F_{\alpha}^{*}(s)$, so

$$
A_{\alpha}^{*}(u)=\operatorname{Res}\left(F_{\alpha}, 0\right)+\sum_{|n|=1}^{N} \operatorname{Res}\left(F_{\alpha}, i \gamma_{n}\right) e^{i \gamma_{n} u} .
$$


Finally, let $B_{\alpha}^{*}(u)=B_{\alpha, T}^{*}(u)$ denote the smoothed trigonometric polynomial defined by

$$
B_{\alpha}^{*}(u)=\operatorname{Res}\left(F_{\alpha}, 0\right)+2 \operatorname{Re} \sum_{0<\gamma_{n}<T}\left(1-\frac{\gamma_{n}}{T}\right) \operatorname{Res}\left(F_{\alpha}, i \gamma_{n}\right) e^{i \gamma_{n} u} .
$$

Ingham established the following theorem for the case $\alpha=0$, and we adapt it here to the broader setting. We use the notation above.

Theorem 3.2. Let $\alpha \in[0,1]$ and $T>1$ be fixed real numbers. Then

$$
\liminf _{u \rightarrow \infty} A_{\alpha}(u) \leq \liminf _{u \rightarrow \infty} B_{\alpha}^{*}(u) \leq B_{\alpha}^{*}(v) \leq \limsup _{u \rightarrow \infty} B_{\alpha}^{*}(u) \leq \limsup _{u \rightarrow \infty} A_{\alpha}(u),
$$

for any positive real number $v$.

Proof. We establish the rightmost inequality; the leftmost one follows from a symmetric argument. Fix a number $\alpha \in[0,1]$ and a real number $T>1$. We may suppose that $\lim \sup _{u \rightarrow \infty} A_{\alpha}(u)<\infty$, since otherwise there is nothing to show, and we may further assume that this value is negative by adding an appropriate constant to both $B_{\alpha}^{*}(u)$ and $A_{\alpha}(u)$. Thus, we assume that $A_{\alpha}(u)<0$ for $u>u_{0}$. Define

$$
D_{\alpha}(s)=F_{\alpha}(s)-F_{\alpha}^{*}(s) .
$$

Since $F_{\alpha}^{*}(s)$ and $D_{\alpha}(s)$ are both regular along the positive real axis, the function $F_{\alpha}(s)$ is as well, so by Landau's theorem the integral in (2.6) converges and this equation holds for $\sigma>0$. Thus

$$
D_{\alpha}(s)=\int_{0}^{\infty} A_{\alpha}(u) e^{-s u} d u-\int_{0}^{\infty} A_{\alpha}^{*}(u) e^{-s u} d u
$$

for $\sigma>0$. Now define

$$
k(t)=k_{T}(t)= \begin{cases}1-|t| / T & \text { if }|t|<T \\ 0 & \text { if }|t| \geq T\end{cases}
$$

and let

$$
K(v)=K_{T}(v)=\frac{4 \sin ^{2}(T v / 2)}{T v^{2}},
$$

so that $\int_{-\infty}^{\infty} k(t) e^{-i t v} d t=K(v)$ and $\int_{-\infty}^{\infty} K(v) e^{i t v} d v=2 \pi k(t)$. Multiplying (3.3) by $k(t) e^{i t w}$ with $w>0$ and integrating in $t$ over $[-T, T]$,

$$
\begin{aligned}
\int_{-T}^{T} D_{\alpha}(\sigma+i t) k(t) e^{i t w} d t= & \int_{0}^{\infty} A_{\alpha}(u) K(u-w) e^{-\sigma u} d u \\
& -\int_{0}^{\infty} A_{\alpha}^{*}(u) K(u-w) e^{-\sigma u} d u
\end{aligned}
$$

for $\sigma>0$, after switching the order of integration in the latter two integrals. (The integrals in (3.3) are uniformly convergent for $t$ in this range for each fixed $\sigma>0$.) 
Since $D_{\alpha}(s)$ is continuous over $\sigma \geq 0$ and $t \in[-T, T]$, the left-hand side of (3.4) is a continuous function of $\sigma$ for $\sigma \geq 0$. Also, for fixed $T$ and $w$, the last integrand in (3.4) is $O\left(u^{-2}\right)$ as $u \rightarrow \infty$, so this integral is absolutely convergent at $\sigma=0$, thus also continuous in $\sigma$ for $\sigma \geq 0$. The first integrand on the right-hand side of (3.4) is nonpositive for $u>u_{0}$, so this integral either diverges to $-\infty$ as $\sigma \rightarrow 0+$, or is continuous on $\sigma \geq 0$, and the first possibility cannot occur due to the behaviour of the other two integrals. Therefore, we may set $\sigma=0$ in (3.4) to obtain

$$
\int_{-T}^{T} D_{\alpha}(i t) k(t) e^{i t w} d t=\int_{0}^{\infty} A_{\alpha}(u) K(u-w) d u-\int_{0}^{\infty} A_{\alpha}^{*}(u) K(u-w) d u .
$$

Now let $w \rightarrow \infty$. Certainly the left-hand side of this equation tends to 0 by the Riemann-Lebesgue lemma, and so

$$
\int_{0}^{\infty} A_{\alpha}(u) K(u-w) d u=\int_{0}^{\infty} A_{\alpha}^{*}(u) K(u-w) d u+o(1)
$$

as $w \rightarrow \infty$. Next, for any $y \in(0, w)$, we see that

$$
\begin{aligned}
\int_{0}^{\infty} A_{\alpha}(u) K(u-w) d u \leq & \left(\sup _{-w \leq v \leq y-w} K(v)\right) \int_{0}^{y}\left|A_{\alpha}(u)\right| d u \\
& +\left(\sup _{u \geq y} A_{\alpha}(u)\right) \int_{y-w}^{\infty} K(v) d v .
\end{aligned}
$$

Letting $w \rightarrow \infty$, then $y \rightarrow \infty$, we conclude that

$$
\limsup _{w \rightarrow \infty} \int_{0}^{\infty} A_{\alpha}(u) K(u-w) d u \leq \limsup _{u \rightarrow \infty} A_{\alpha}(u) \int_{-\infty}^{\infty} K(v) d v=2 \pi \limsup _{u \rightarrow \infty} A_{\alpha}(u) .
$$

For convenience, define $\gamma_{0}=0$, and let $r_{n}=\operatorname{Res}\left(F_{\alpha}, i \gamma_{n}\right)$ for each integer $n$. We then see that as $w \rightarrow \infty$,

$$
\begin{aligned}
\int_{0}^{\infty} A_{\alpha}^{*}(u) K(u-w) d u & =\sum_{n=-N}^{N} r_{n} e^{i \gamma_{n} w} \int_{-w}^{\infty} K(v) e^{i \gamma_{n} v} d v \\
& =2 \pi \sum_{n=-N}^{N} r_{n} e^{i \gamma_{n} w} k\left(\gamma_{n}\right)+o(1) \\
& =2 \pi B_{\alpha}^{*}(w)+o(1),
\end{aligned}
$$

and the inequality follows.

Finally, let $v$ be a fixed positive real number. By Dirichlet's theorem on simultaneous approximation, there exist infinitely many positive integers $q=u-v$ such that $\gamma_{n} u$ is as close as we desire to $\gamma_{n} v(\bmod 2 \pi)$, for each $\gamma_{n}<T$. This implies that $\lim \inf _{u \rightarrow \infty} B_{\alpha}^{*}(u) \leq B_{\alpha}^{*}(v) \leq \lim \sup _{u \rightarrow \infty} B_{\alpha}^{*}(u)$. 
We remark that this proof would not seem to apply in the case where $\alpha=1 / 2$ if we were to replace $A_{1 / 2}(u)$ by $L_{1 / 2}\left(e^{u}\right)$, due to the pole of order two at the origin in its Laplace transform.

Ingham also proved that if $L_{0}(x) / \sqrt{x}$ is either bounded above by a constant or bounded below by a constant, then there exist infinitely many linear dependencies over $\mathbb{Z}$ of the imaginary parts of the zeros of the zeta function lying in the upper half plane. We show that this statement has a natural analogue for the cases $\alpha \in[0,1]$.

Theorem 3.3. Let $\alpha \in[0,1]$. If the imaginary parts $\gamma_{1}<\gamma_{2}<\cdots$ of the distinct zeros of $\zeta(s)$ lying above the real axis are connected by at most a finite number of linear relations of the type $\sum_{n=1}^{N} c_{n} \gamma_{n}=0$, where the $c_{n}$ are integers, not all zero, then

$$
\liminf _{x \rightarrow \infty} \mathcal{L}_{\alpha}(x) x^{\alpha-\frac{1}{2}}=-\infty \quad \text { and } \quad \limsup _{x \rightarrow \infty} \mathcal{L}_{\alpha}(x) x^{\alpha-\frac{1}{2}}=\infty .
$$

Proof. In view of Theorem 2.4, we may assume the Riemann hypothesis and the simplicity of the zeros of the zeta function. Select $S$ so that no nontrivial integer relations exist among the $\gamma_{n}$ with $\gamma_{n}>S$, and define $C(S)$ by

$$
C(S)=2 \sum_{0<\gamma_{n} \leq S}\left|\operatorname{Res}\left(F_{\alpha}, i \gamma_{n}\right)\right| .
$$

Select $T>2 S$. Since the $\gamma_{n}$ with $S<\gamma_{n}<T$ are linearly independent, by Kronecker's theorem on simultaneous approximation there exist arbitrarily large values of $u$ for which $\gamma_{n} u(\bmod 2 \pi)$ is as close as we desire to $-\arg \left(\operatorname{Res}\left(F_{\alpha}, i \gamma_{n}\right)\right)$, for each $\gamma_{n} \in(S, T)$. Therefore,

$$
\begin{aligned}
\limsup _{u \rightarrow \infty} B_{\alpha}^{*}(u) & \geq \operatorname{Res}\left(F_{\alpha}, 0\right)-C(S)+2 \sum_{S<\gamma_{n}<T}\left(1-\frac{\gamma_{n}}{T}\right)\left|\operatorname{Res}\left(F_{\alpha}, i \gamma_{n}\right)\right| \\
& \geq \operatorname{Res}\left(F_{\alpha}, 0\right)-C(S)+\sum_{S<\gamma_{n}<T / 2}\left|\operatorname{Res}\left(F_{\alpha}, i \gamma_{n}\right)\right|,
\end{aligned}
$$

and this is unbounded as $T$ grows large by Lemma 3.1. By Theorem 3.2, we conclude that $\lim _{\sup _{u \rightarrow \infty}} A_{\alpha}(u)=\infty$, and this establishes the latter conclusion of the theorem. The former part may be proved in a similar manner.

We remark that there appears to be little numerical or heuristic evidence for linear dependencies among the $\gamma_{n}$ (see [8]).

\section{Formulas and problems}

Throughout this section we assume the Riemann hypothesis as well as the simplicity of the zeros of the zeta function. Using these assumptions together with Perron's formula (2.1), we obtain an asymptotic estimate for $\mathcal{L}_{\alpha}(x)$ for each $\alpha$ in [0,1]. Again, we denote the complex zeros of $\zeta(s)$ in the upper half plane by $\rho_{n}=\frac{1}{2}+i \gamma_{n}$, with $\left\{\gamma_{n}\right\}$ an increasing sequence. 
If $\alpha \in[0,1]$, we move the line of integration in (2.1) to the left to $\frac{1}{2}-\alpha-\delta \pm i T$, for some small positive $\delta$. Employing the residues listed in (3.1) and (3.2), and arguing as in the proof of Theorem 2.1, we find that if $\alpha \neq 1 / 2$ then

$$
\mathcal{L}_{\alpha}(x)=x^{\frac{1}{2}-\alpha}\left(\frac{1}{(1-2 \alpha) \zeta\left(\frac{1}{2}\right)}+\sum_{\left|\gamma_{n}\right|<T} \frac{\zeta\left(2 \rho_{n}\right) x^{i \gamma_{n}}}{\zeta^{\prime}\left(\rho_{n}\right)\left(\rho_{n}-\alpha\right)}\right)+E(x, T),
$$

where $E(x, T)$ denotes an error term that tends to 0 as $T \rightarrow \infty$, and

$$
\mathcal{L}_{1 / 2}(x)=\frac{\gamma}{\zeta\left(\frac{1}{2}\right)}-\frac{\zeta^{\prime}\left(\frac{1}{2}\right)}{2 \zeta\left(\frac{1}{2}\right)^{2}}+\sum_{\left|\gamma_{n}\right|<T} \frac{\zeta\left(2 \rho_{n}\right) x^{i \gamma_{n}}}{i \gamma_{n} \zeta^{\prime}\left(\rho_{n}\right)}+E(x, T) .
$$

In the former case, the sum in (4.1) is highly oscillatory, so one would expect the constant term $1 /(1-2 \alpha) \zeta(1 / 2)$ to dominate in this expression for most values of $x$. When $\alpha=0$, this term is negative, and when $\alpha=1$ it is positive, so we can see the cause of the predisposition of negative terms in $L_{0}(x)$, and of positive ones in $L_{1}(x)$. Likewise, in (4.2) the constant term is $0.5244 \ldots>0$, so we expect $\mathcal{L}_{1 / 2}(x)$ to be skewed toward positive values. We can therefore state two problems that generalise the questions of Pólya and Turán directly.

Problem 4.1. For each $\alpha \in[0,1]$, show that $\mathcal{L}_{\alpha}(x)$ changes sign infinitely often.

Problem 4.2. For each $\alpha \in[0,1 / 2)$, determine the smallest nontrivial value of $x$ where $\mathcal{L}_{\alpha}(x)>0$, and for $\alpha \in[1 / 2,1]$, determine the smallest positive value of $x$ where $\mathcal{L}_{\alpha}(x)<0$.

Naturally, we expect that sign changes do indeed exist, since we have shown that sign constancy in any of these functions would imply not only the Riemann hypothesis and the simplicity of the zeros of the zeta function, but also the existence of infinitely many dependency relations among the zeros of the zeta function in the upper half plane.

As described in the introduction, prior investigations [1, 3, 9] have resolved these two problems for the cases $\alpha=0$ and $\alpha=1$ (and thus, by continuity, also for small intervals containing these values). The case $\alpha=1 / 2$ may also be handled easily: we compute that the function $\mathcal{L}_{1 / 2}(x)$ attains its first negative value at $x=32$. This function is indeed positively skewed: it is negative at just eight other integers less than 1000 (namely, 80, 200, 286, 288, 290, and 292-294), and at fewer than 545000 different positive integers below $10^{8}$. Also, we calculate that $B_{1 / 2}^{*}(\log 1132)=-0.0223 \ldots$ when $T=2000$, so $\mathcal{L}_{1 / 2}(x)<0$ at infinitely many integers $x$ by Theorem 3.2.

Theorem 3.2 may presumably be employed in a similar way to establish the existence of sign changes in $\mathcal{L}_{\alpha}(x)$ for other $\alpha \in[0,1]$. However, since $1 /|(1-2 \alpha) \zeta(1 / 2)|$ increases as $\alpha \rightarrow 1 / 2$, we expect that this strategy will be more difficult as $\alpha$ approaches $1 / 2$. Likewise, we expect that resolving Problem 4.2 for additional values of $\alpha$ by explicit calculation as in [1] will be much more difficult for $\alpha$ near $1 / 2$. 


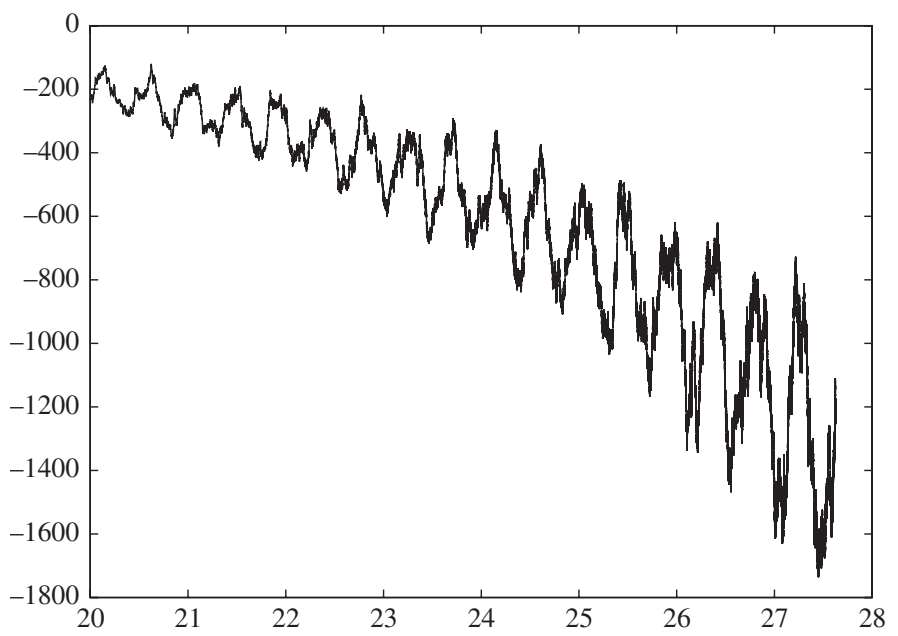

FIGURE 1. $L_{1 / 4}\left(e^{u}\right)$ for $20 \leq u \leq 12 \log 10$.

The case $\alpha=1 / 2$ presents some additional questions. By Theorem 2.1, the function $L_{\alpha}(x)$ converges to the negative real number $\zeta(2 \alpha) / \zeta(\alpha)$ for $1 / 2<\alpha<1$ under the Riemann hypothesis, and so $L_{\alpha}(x)<0$ for sufficiently large $x$ under this assumption when $\alpha \in(1 / 2,1)$. It is then natural to ask if the Riemann hypothesis implies that $L_{1 / 2}(x)$ is also eventually negative. Also, by Theorem 2.4, sign constancy in $L_{1 / 2}(x)=\mathcal{L}_{1 / 2}(x)+(\log x) / 2 \zeta(1 / 2)$ implies the Riemann hypothesis and a bound on the multiplicity of the zeros of the zeta function, but it does not appear to violate the linear independence criterion. We therefore propose the following two natural problems. The first is very similar to the questions of Pólya and Turán.

Problem 4.3. Determine if $L_{1 / 2}(x) \leq 0$ for all $x \geq 17$.

Problem 4.4. Does the Riemann hypothesis imply that $L_{1 / 2}(x) \leq 0$ for sufficiently large $x$ ?

The next section describes a computational technique to investigate Problems 4.2 and 4.3 .

\section{Computational investigations}

We employ the method of [1] to investigate the behaviour of the functions $L_{\alpha}(x)$ for $\alpha=1 / 4, \alpha=1 / 2$, and $\alpha=3 / 4$ over a large interval. This method adopts a sieving procedure to compute the values of the Liouville function across successive intervals. We briefly describe the method here. First, we construct a table of values for $\lambda(n)$ for $n<N$, where $N$ is a parameter of the algorithm. Rather than storing all $N$ values of the Liouville function, however, it is more efficient to store only the value of $\lambda(n)$ where $\operatorname{gcd}(n, 30)=1$, so that only $\varphi(30)=8$ bits are needed for each block of 30 integers. 


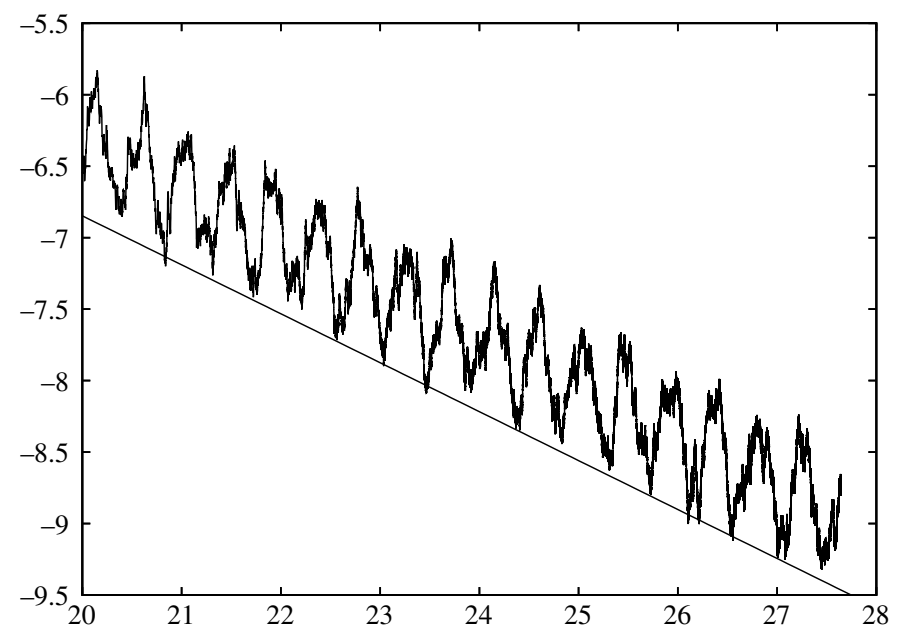

FigURE 2. $L_{1 / 2}\left(e^{u}\right)$ for $20 \leq u \leq 12 \log 10$, and $u / 2 \zeta(1 / 2)$.

Second, we arrange the remaining integers we wish to test into successive blocks $[a, b]$ of size $M$, where $M$ is another parameter of the method. Each of these blocks is then sieved for prime factors in three main stages. In the first stage, we look for multiples of large primes $p$, which satisfy $b /(N-1) \leq p \leq \sqrt{b}$. A multiple $k p$ of such a prime has $k<N$, and so our table may be used to determine $\lambda(k p)=-\lambda(k)$, once multiples of 2,3 , and 5 are taken into account. In the next stage, we sieve with the primes from $\lceil b /(N-1)\rceil-1$ down to 7 , and whenever a multiple $k p$ of such a prime $p$ is detected and $\lambda(k p)$ is not yet known, we continue to factor $k$ until we find a factor $\ell$ of $k$ with $\ell<N$. We may then use our table to determine $\lambda(\ell)$, and hence $\lambda(k p)$. After this, any integers in the interval not yet accounted for have the form $2^{a} 3^{b} 5^{c} q$, where $a, b$ and $c$ are nonnegative integers, and $q$ is either 1 or a prime greater than $\sqrt{b}$. These values are detected and the values of the Liouville function computed accordingly. We then use these values of $\lambda(n)$ to determine the weighted sums of the Liouville function for certain $\alpha$ across this interval. Intervals were sieved independently across several computers on a cluster at the Centre for Interdisciplinary Research in the Mathematical and Computational Sciences (IRMACS) at Simon Fraser University, and the results then combined to determine values of $L_{\alpha}(x)$ across the entire range.

We set $N=55 \cdot 10^{9}+20$, so that our table required approximately $1.7 \mathrm{~GB}$ of storage, and set $M=2 \cdot 10^{7}$. We then used this method to compute $L_{\alpha}(x)$ with $\alpha=1 / 4$, $\alpha=1 / 2$, and $\alpha=3 / 4$ for $x \leq 10^{12}$, using GMP [2] to compute each summand to 256 bits of precision. The positive and negative terms in each sum were accumulated separately to avoid cancellation issues. We recorded the value of each of these sums at regular intervals, and we exhibit the resulting plots of $L_{\alpha}\left(e^{u}\right)$ in Figures $1-3$. Figures 2 and 3 also exhibit the adjustment needed to create $\mathcal{L}_{\alpha}\left(e^{u}\right)$ for $\alpha=1 / 2$ and $\alpha=3 / 4$ by using (2.3), so $u / 2 \zeta(1 / 2)$ in the former case and $\zeta(3 / 2) / \zeta(3 / 4)$ in the latter. Our 


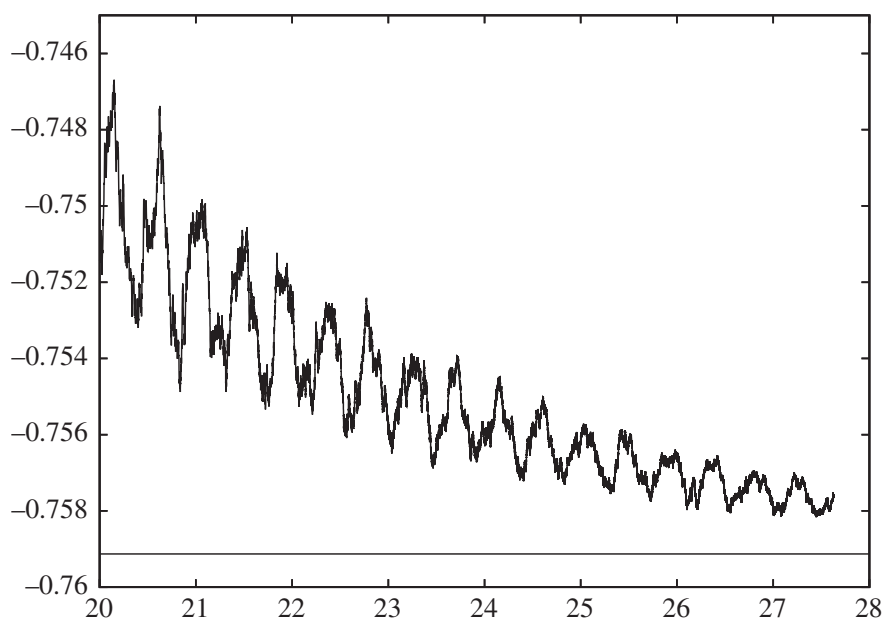

FIGURE 3. $L_{3 / 4}\left(e^{u}\right)$ for $20 \leq u \leq 12 \log 10$, and $\zeta(3 / 2) / \zeta(3 / 4)$.

calculations verified that $L_{1 / 4}(x)<0$ for $11 \leq x \leq 10^{12}$, that $L_{1 / 2}(x)<0$ for $17 \leq x \leq$ $10^{12}$, and that $L_{3 / 4}(x)>\zeta(3 / 2) / \zeta(3 / 4)$ for $1 \leq x \leq 10^{12}$. In fact, the minimum value of $L_{3 / 4}(x)$ over this range is $-0.7581617368 \ldots$, occurring at $x=835018639060$, while $\zeta(3 / 2) / \zeta(3 / 4)=-0.7591277836 \ldots$ Thus, any nontrivial sign crossings in Problems 4.2 or 4.3 for these values of $\alpha$ must occur for $x>10^{12}$.

\section{Acknowledgements}

We are grateful to Richard Brent, Michael Coons, and Amir Akbary for discussions on this problem. We thank the IRMACS Centre at Simon Fraser University for computational resources. We also thank the referee for many valuable comments that greatly helped to shape the final form of this paper.

\section{References}

[1] P. Borwein, R. Ferguson and M. J. Mossinghoff, 'Sign changes in sums of the Liouville function', Math. Comp. 77(263) (2008), 1681-1694.

[2] GMP: The GNU multiple precision arithmetic library. http://gmplib.org.

[3] C. B. Haselgrove, 'A disproof of a conjecture of Pólya', Mathematika 5 (1958), 141-145.

[4] A. E. Ingham, 'On two conjectures in the theory of numbers', Amer. J. Math. 64 (1942), 313-319.

[5] R. S. Lehman, 'On Liouville's function', Math. Comp. 14 (1960), 311-320.

[6] M. R. Murty, Problems in Analytic Number Theory, 2nd edn, Graduate Texts in Mathematics, 206 (Springer, New York, 2008).

[7] G. Pólya, 'Verschiedene Bemerkungen zur Zahlentheorie', Jahresber. Deutsch. Math.-Verein. 28 (1919), 31-40.

[8] M. Rubinstein and P. Sarnak, 'Chebyshev's bias', Experiment. Math. 3(3) (1994), 173-197.

[9] M. Tanaka, 'A numerical investigation on cumulative sum of the Liouville function', Tokyo J. Math. 3(1) (1980), 187-189. 
[10] E. C. Titchmarsh, The Theory of the Riemann Zeta-Function, 2nd edn (Oxford University Press, New York, 1986).

[11] P. Turán, 'On some approximative Dirichlet-polynomials in the theory of the zeta-function of Riemann', Danske Vid. Selsk. Mat.-Fys. Medd. 24(17) (1948), 1-36.

[12] P. Turán, 'Nachtrag zu meiner Abhandlung "On some approximative Dirichlet polynomials in the theory of zeta-function of Riemann”", Acta Math. Acad. Sci. Hungar. 10 (1959), 277-298.

MICHAEL J. MOSSINGHOFF,

Department of Mathematics, Davidson College,

Davidson, NC, 28035-6996, USA

e-mail: mimossinghoff@davidson.edu

TIMOTHY S. TRUDGIAN,

Department of Mathematics and Computer Science,

University of Lethbridge, Alberta, Canada T1K 3M4

e-mail: tim.trudgian@uleth.ca 